УДК 622.4

\title{
ЭФФЕКТИВНОСТЬ ПРОВЕТРИВАНИЯ ТУПИКОВЫХ ПОДГОТОВИТЕЛЬНЫХ ВЫРАБОТОК ПОСЛЕ ВЗРЫВНЫХ РАБОТ
}

\author{
Колесов Евгений Викторович1, \\ kolesovev@gmail.com \\ Казаков Борис Петрович1, \\ aero_kaz@mail.ru \\ 1 Горный институт УрО РАН, \\ Россия, 614007, г. Пермь, ул. Сибирская, 78-А.
}

\begin{abstract}
Актуальность исследования обусловлена необходимостью улучшения системы вентиляции тупиковых выработок, сокращения трудозатрат на их вентиляцию и ускорения выполнения основных операций по проходке выработок.

Цель: определение оптимального расстояния от конца трубопровода до груди забоя тупиковой выработки с точки зрения эфффективности проветривания тупиковой подготовительной выработки.

объект: система вентиляции тупиковой подготовительной выработки.

Методы: многопараметрическое трехмерное численное моделирование на основе методов вычислительной гидродинамики. Приведены результаты трехмерного численного моделирования проветривания тупиковой подготовительной выработки от выделившихся в результате взрывных работ ядовитых газов. Варьируемыми параметрами модели являются: начальная концентрация и распределение вредных примесей в тупиковой выработке после проведения взрывных работ, расстояние от кониа нагнетательного трубопровода до тупикового забоя, поперечное сечение тупиковой выработки, а также расход свежего воздуха, поступающего через трубопровод. Получена расчетная формула для определения объема зоны смешения. Показано, что время проветривания тупиковой выработки не зависит от длины зоны отброса газов, а зависит от начальной средней концентрации ядовитых газов в зоне смешения. Получены значения безразмерного коэффрициента эфффективности проветривания К тупиковой выработки, или коэфффициента турбулентной дифффузии В.Н. Воронина, в зависимости от варьируемых параметров. Показано, что коэфффициент $\mathrm{K}$ не зависит от величины входящего расхода свежего воздуха, а зависит только от геометрических параметров системы вентиляции тупиковой выработки и принимает постоянное значение для данной консфигурации системы вентиляции. Показано, что существует оптимальное расстояние от конца трубопровода до забоя, при котором происходит наиболее эфрфективный вынос вредных примесей.
\end{abstract}

\section{Ключевые слова:}

Взрывные работы, CFD-моделирование, тупиковая выработка, эфффективность вентиляции, зона смешения, концентрация газов.

\section{Введение}

При проходке подземных горных выработок буровзрывным способом в результате взрыва в области вблизи забоя образуется зона с повышенной концентрацией ядовитых газов (как правило, $\mathrm{CO}$ и $\mathrm{NO}_{\mathrm{x}}$ ) [1] Допуск людей в забой после взрывных работ разрешается производить только после того, как концентрация ядовитых газов в тупиковой выработке станет меньше предельно допустимой: менее 0,0017 \% по объему для $\mathrm{CO}$ и менее 0,00026 \% по объему для $\mathrm{NO}_{\mathrm{x}}$ в пересчете на $\mathrm{NO}_{2}$. При этом согласно «Правилам безопасности при ведении горных работ и переработке твердых полезных ископаемых», действующим на территории России, тупиковые горные выработки длиной более 10 м должны проветриваться активной струей воздуха, подаваемой по вентиляционному трубопроводу при помощи вентилятора местного проветривания (ВМП). Дополнительным условием является то, что расстояние от конца нагнетательного трубопровода до тупикового забоя не должно превышать 10 м. Данное требование приводит к дополнительным трудозатратам, связанным с постоянным снятием и установкой трубопровода до и после взрывных работ, поскольку иначе воздействие ударной волны и разлетающихся при взрыве кусков горной породы нарушит целостность трубопровода, что потребует замены его концевой части протя- женностью до 20-30 м. Увеличение максимального расстояния от конца трубопровода до забоя позволит сократить трудозатраты на монтаж трубопровода и ускорит выполнение основных операций по проходке выработки. Поэтому представляет интерес исследование эффективности выноса вредных газов в зависимости от расстояния от конца трубопровода до забоя и других параметров вентиляции тупиковой горной выработки. Таким образом, цель настоящего исследования - определение оптимального расстояния от конца трубопровода до груди забоя тупиковой выработки с точки зрения эффективности проветривания тупиковой подготовительной выработки.

Благодаря развитию вычислительных технологий в настоящее время возможно исследование характеристик аэродинамических потоков с помощью специализированных пакетов математического моделирования методами вычислительной гидродинамики (CFD - computational fluid dynamics). В современной литературе представлено множество работ, связанных с применением методов CFD для решения задач рудничной вентиляции [2-20]. В работе [2] рассмотрены основные принципы CFD и обсуждены шесть широко используемых моделей турбулентности с некоторыми примерами их применения и рекомендациями по выбору подходящей модели турбулентности. Авторы 
работы [3] исследовали эффективность проветривания тупиковых выработок с помощью продольных перегородок на основе CFD-моделирования. В статье [4] строится CFD-модель вентиляции реальной выработки после взрывных работ и производится верификация модели путем сопоставления результатов моделирования и натурных данных. В [5] описывается проведение натурных замеров концентрации ядовитых газов после взрывных работ в тупиковой выработке, оценивается длина зоны отброса газов и время проветривания выработки до ПДК. В [6] исследуется динамика пылевоздушной смеси при проветривании тупиковой выработки в процессе работы комбайново- го комплекса. В [7] исследуется возможность последовательного проветривания нескольких тупиковых проходческих выработок после взрывных работ.

\section{Постановка задачи}

Исследуется тупиковая подготовительная выработка в период после проведения взрывных работ, проветриваемая струей свежего воздуха, подаваемого по вентиляционному трубопроводу, или воздуховоду, с помощью ВМП, расположенного в сквозной выработке на свежей струе воздуха. Схема проветривания тупиковой выработки представлена на рис. 1.

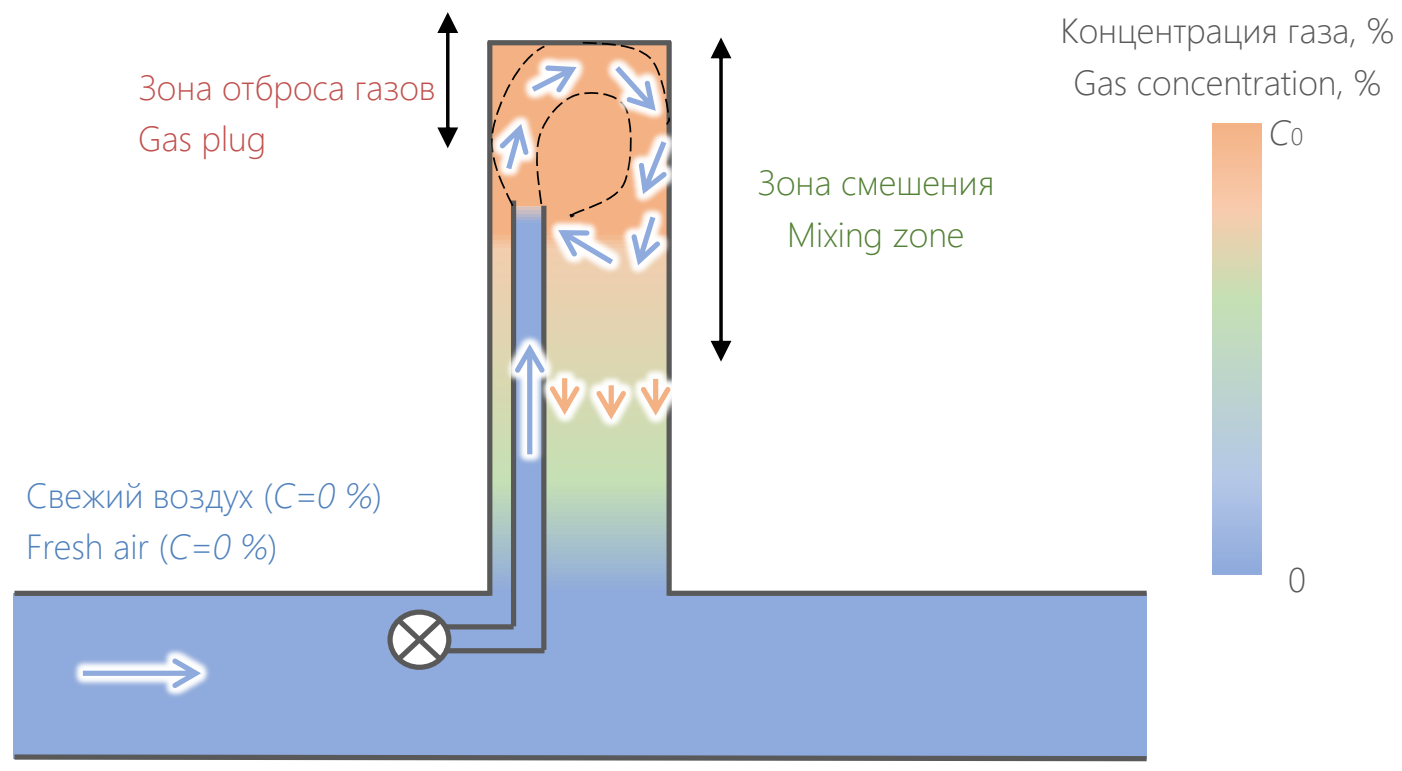

Pис. 1. Схема проветривания тупиковой выработки после взрывных работ

Fig. 1. Scheme of dead-end heading ventilation after blasting operations

Таблица 1. Формулы для оченки длины зоны отброса газов

Table 1. Gas plug length estimation formulas

\begin{tabular}{|c|c|}
\hline Автор/Author & Формула/Formula \\
\hline $\begin{array}{l}\text { Ксенофонтова А.И. } \\
\text { Ksenofontova A.I. }\end{array}$ & $l=15+A / 5$ \\
\hline $\begin{array}{l}\text { Bopoнин B.H. } \\
\text { Voronin V.N. }\end{array}$ & $l=\frac{20 \mathrm{~A}}{\rho l_{\amalg} \sqrt{S}}$ \\
\hline $\begin{array}{l}\text { Мостепанова Ю.Б., Русак О.Н. } \\
\text { Mostepanova Yu.B., Rusak O.N. }\end{array}$ & $l=\frac{11,5 A}{S}$ \\
\hline $\begin{array}{l}\text { Комаров В.Б., Килькеев Ш.Х. } \\
\text { Komarov V.B., Kilkeev Sh.Kh. }\end{array}$ & $l=10+2,4 \mathrm{~A}$ \\
\hline $\begin{array}{l}\text { Сметанин М.М., } \\
\text { Барышев А.С., Хохлов Н.А. } \\
\text { Smetanin M.M., } \\
\text { Barishev A.S., Khokhlov N.A. }\end{array}$ & $l=\frac{16,5 \sqrt{A}}{\sqrt[3]{S}}$ \\
\hline $\begin{array}{l}\text { Янов А.П., Ващенко В.C. } \\
\text { Yanov A.P., Vashchenko V.S. }\end{array}$ & $l=\frac{N}{l_{\amalg}}+0,3 \mathrm{~A}$ \\
\hline $\begin{array}{l}\text { Барышев А.С., Казаков А.П. } \\
\text { Baryshev A.S., Kazakov A.P. }\end{array}$ & $l=0,5 k_{1} \sqrt{S}\left(1+\frac{1}{2 a}\right)+\frac{A b}{S}$ \\
\hline
\end{tabular}

Согласно проведенным исследованиям $[1,8]$ после взрывных работ в призабойной части тупиковой выработки образуется зона отброса газов с временным превышением предельно допустимой концентрации (ПДК) вредных газов $\mathrm{CO}$ и $\mathrm{NO}_{x}$. В настоящее время существует множество эмпирических формул, по которым рассчитывается длина зоны отброса газов. В табл. 1 приведены эмпирические формулы, предложенные разными авторами на основании различных исследований [8].

В приведенных формулах $l$ - длина зоны отброса газов, м; $A$ - масса одновременно взрываемого взрывчатого вещества (BВ), кг; $S$ - площадь поперечного сечения выработки, м ${ }^{2} ; \rho$ - плотность взрываемых

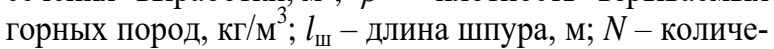
ство одновременно взрываемых шпуров; $k_{1}$ - безразмерный коэффициент, учитывающий крепость горных пород и конструкцию заряда ВВ (для крепости пород 2-5 значение коэффициента близко к единице); $a$ - коэффициент турбулентной структуры свободных струй (значение лежит в пределах $0,08-0,14) ; b$ - газовость ВВ, $\mathrm{M}^{3} /$ кг.

Следует отметить, что значения длины зоны отброса газов для одной и той же выработки, посчитанные по этим формулам, могут отличаться в несколько раз, а в некоторых случаях значения могут иметь отличия в несколько порядков, и поэтому применимость указанных формул нужно определять в каждом конкретном 
случае. В связи с этим в настоящей работе был принят другой подход для исследования эволюции средней концентрации примеси в тупиковой выработке.

При нагнетательном способе проветривания тупиковой выработки в объеме вблизи забоя создается вихревая область с разнонаправленным движением воздуха, в которой происходит перемешивание чистого и загазованного воздуха за счет интенсивного перемешивания воздушных потоков, движущихся в режиме развитой турбулентности. В настоящей работе эта область называется зоной смешения и рассчитывается как объем выработки, ограниченный грудью забоя и сечением выработки, после которого движение воздуха всюду направлено к устью выработки. Таким образом, время, необходимое для проветривания тупиковой выработки после взрывных работ, рассчитывается как сумма времени разбавления концентрации вредных газов до ПДК в зоне смешения и времени однократного воздухообмена в оставшемся объеме проветриваемой выработки.

Эволюция средней концентрации газов $C(t)$ в зоне смешения $V_{3 \mathrm{C}}$ описывается моделью Воронина [1], представляющей уравнение баланса массы примеси в объеме зоны смешения с начальным условием:

$$
\left\{\begin{array}{l}
\frac{d C(t)}{d t}-K \frac{Q}{V_{3 С}} C(t)=0 ; \\
C(0)=C_{0}=\frac{A \cdot b}{V_{3 \mathrm{C}}},
\end{array}\right.
$$

где $K$ - коэффициент эффективности проветривания; $Q$ - расход воздуха, выходящий из воздуховода, $\mathrm{m}^{3} / \mathrm{c}$, задается согласно проекту организации работ или паспорту проветривания; $A$ - масса одновременно взрываемого ВВ, кг, задается согласно проекту или паспорту взрывных работ; $b$ - газовость $\mathrm{BB}, \mathrm{M}^{3} /$ кг, задается согласно техническим условиям (ТУ) на взрывчатое вещество.

Решением системы уравнений (1) является экспоненциальная функция:

$$
C(t)=C_{0} \exp \left(-\frac{K \cdot Q}{V_{3 \mathrm{C}}} t\right) .
$$

В полученном решении фигурируют два неизвестных параметра: коэффициент эффективности проветривания и объём зоны смешения. Для их оценки была разработана трехмерная численная модель проветривания тупиковой выработки на основе методов вычислительной гидродинамики.

\section{Численное моделирование процесса проветривания тупиковой выработки после взрывных работ}

Параметры исходной модели следующие: сечение тупиковой выработки - арочное, площадь сечения $17 \mathrm{~m}^{2}$, длина выработки - 50 м; воздуховод находится вблизи стенки у кровли выработки, диаметр воздуховода - 1 м, скорость воздушной струи на выходе из воздуховода - 10 м/с, расстояние между забоем выработки и концом воздуховода - 10 м (рис. 2).

В модели использовалась модель турбулентности standard $k$ - $\varepsilon$ с масштабируемой пристеночной функцией. После проведения серии тестовых расчетов на независимость решения от плотности сетки было выбрано разбиение расчетной области на сетку, состоящую из 3,8 млн элементов, уплотнением вблизи стенок выработки и воздуховода, шаг по времени 0,5 секунды (рис. 3). Величина безразмерного расстояния от стенки до центра первой ячейки $y^{+}$в среднем составляет около 40, не превышая значения 180.

Полученная модель использовалась в дальнейшем для исследования зависимости времени проветривания тупиковой выработки от трёх параметров: площади сечения выработки $S$, отставания трубопровода от груди забоя $L_{\text {от }}$ расхода воздуха на выходе из воздуховода $Q$. Пространство параметров модели представлено на рис. 4. При исследовании влияния каждого параметра два других фиксировались. Фиксируемые параметры обозначены красным цветом. В общей сложности было разработано и рассчитано 27 конфигураций.

На первом этапе исследования были определены объемы зон смешения для различных конфигураций варьируемых параметров. Получена эмпирическая формула для определения объема зоны смешения:

$$
\begin{gathered}
L_{3 \mathrm{C}}=L_{0 \mathrm{~T}}+4 d_{\Pi}, \\
V_{3 \mathrm{C}}=S \cdot L_{3 \mathrm{C}},
\end{gathered}
$$

где $L_{3 \mathrm{C}}$ - длина зоны смешения, отсчитываемая от забоя выработки, м; $L_{\mathrm{OT}}$ - расстояние от груди забоя до конца вентиляционного трубопровода, м; $d_{\text {п }}$ приведенный диаметр выработки, м; $S$ - сечение выработки, м. $^{2}$.

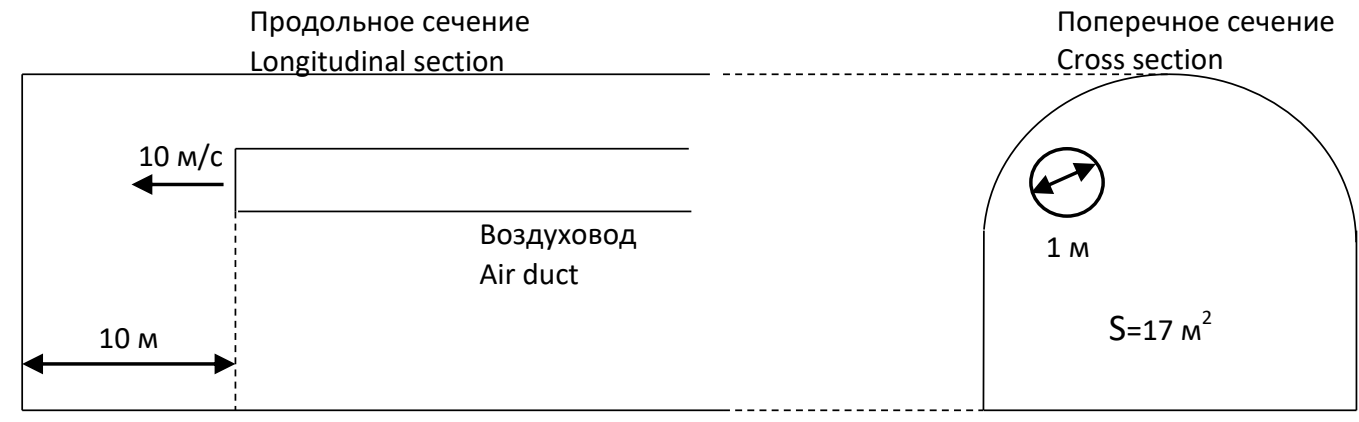

Pис. 2. Схема призабойной части проветриваемой тупиковой выработки

Fig. 2. Scheme of dead-end heading face 


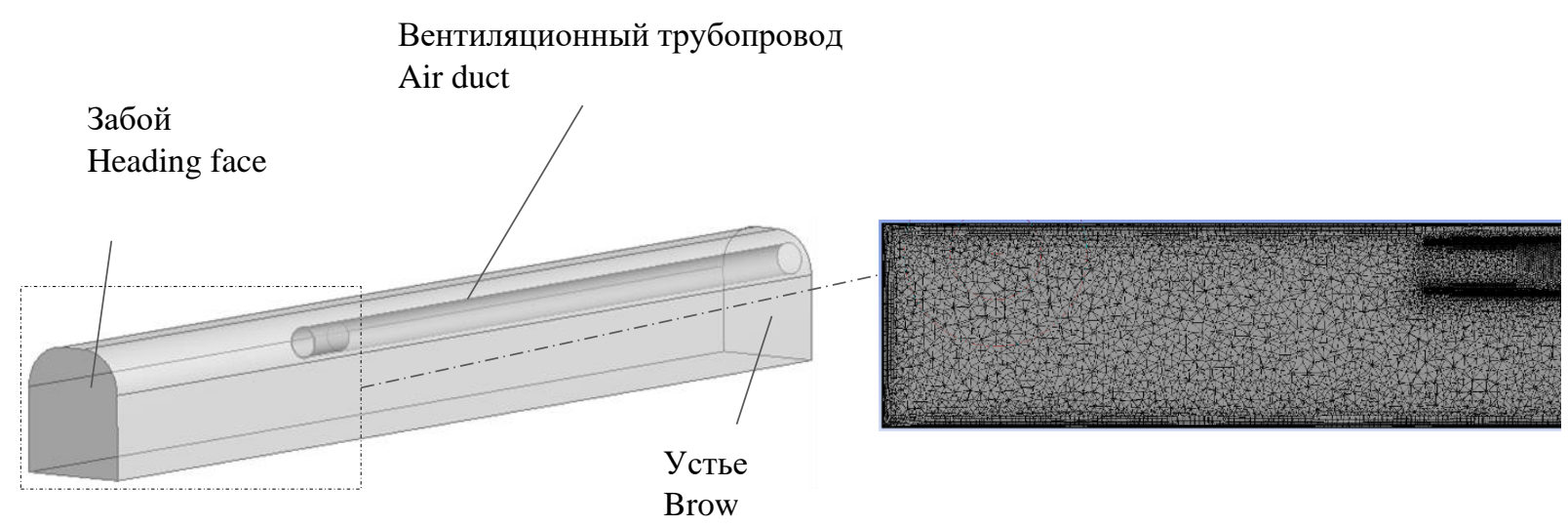

Рис. 3. Геометрия области и расчётная сетка модели тупиковой выработки

Fig. 3. Geometry and meshing of the dead-end heading model

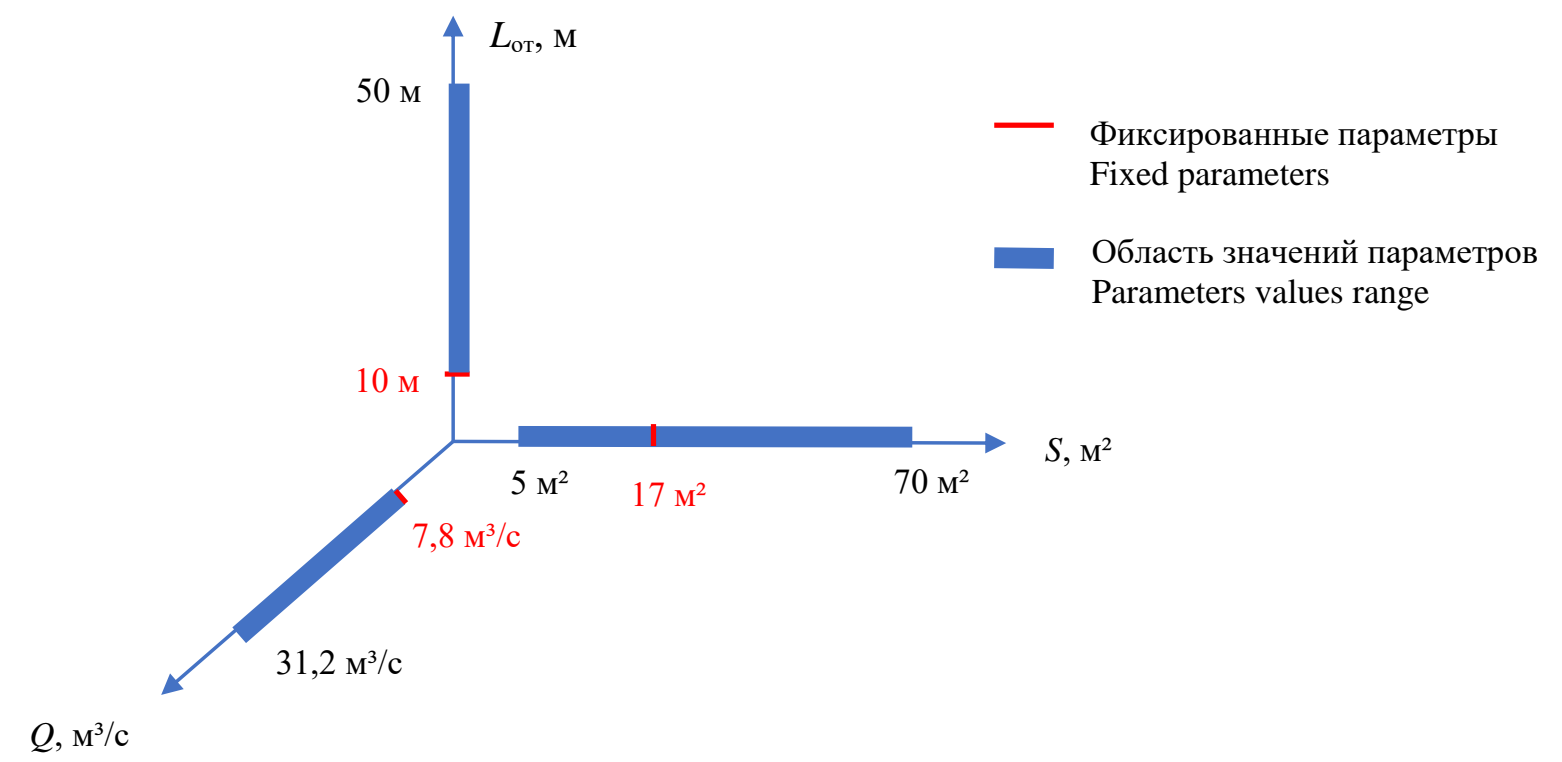

Puc. 4. Пространство параметров модели

Fig. 4. Model parameters

Приведенный диаметр выработки рассчитывается по следующей формуле:

$$
d_{\Pi}=4 \frac{S}{P},
$$

где $P$ - периметр выработки, м.

В каждой модели в начальный момент времени в зоне смешения задавалась начальная средняя концентрация газовой примеси, образующаяся после взрыва ВВ с газовостью $0,022 \mathrm{~m} /$ кг. Удельная масса ВB составляет 19,4 кг/ $\mathbf{M}^{2}$.

Ниже представлены результаты моделирования в один из моментов времени: на рис. 5 - поле концентрации газов в продольном срезе через центр воздуховода, на рис. 6 - линии тока воздуха в расчетной области. В призабойной зоне образуется крупномасштабный вихрь, от формы и размеров которого зависит эффективность выноса вредных примесей, и со- ответственно значение коэффициента эффективности выноса примесей $K$.

\section{Выводы}

1. В результате моделирования во всех исследуемых моделях был подтвержден экспоненциальный закон уменьшения средней концентрации примеси в зоне смешения (2).

2. Для исследования зависимости времени проветривания зоны смешения от длины зоны отброса газов были рассчитаны модели с длиной зоны отброса газов 1 м и длиной, равной длине зоны смешения (при этом массы и газовость ВВ оставались прежние). Времена проветривания зон смешения до ПДК отличались от времён проветривания в исходных моделях в пределах $5 \%$. 

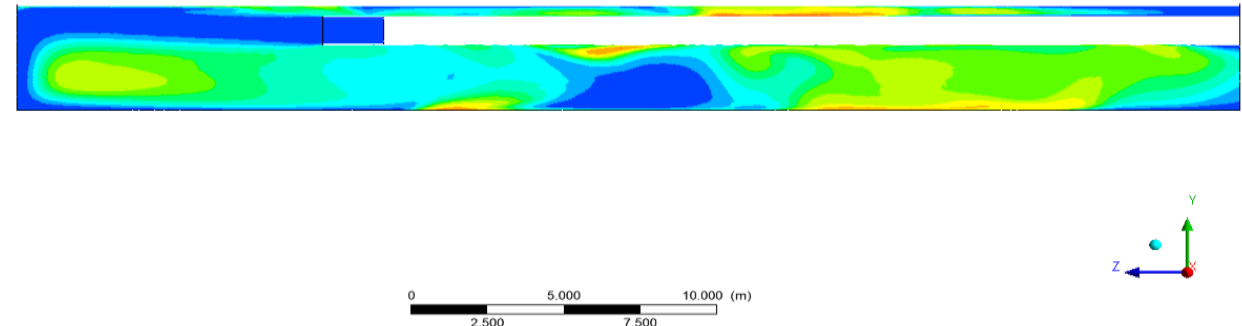

Рис. 5. Поле концентрации газов. Продольное сечение через иентр воздуховода

Fig. 5. Gas concentration field. Longitudinal cross section through the air duct center
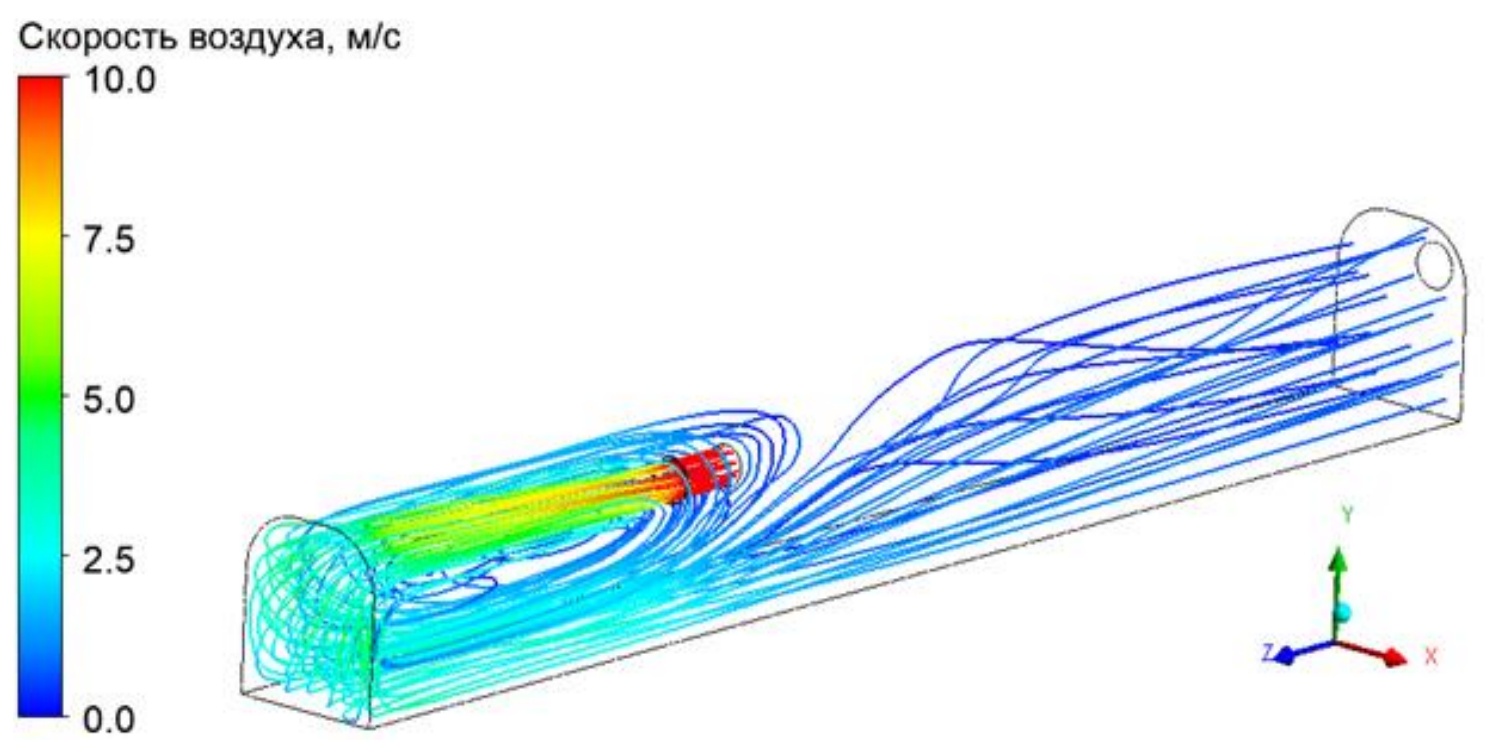

Pис. 6. Линии тока воздуха

Fig. 6. Air streamlines

Таким образом, нет необходимости оценивать длину зоны отброса газов, достаточно считать только начальную среднюю концентрацию в зоне смешения по формуле:

$$
C(0)=\frac{A b}{V_{3 c}} .
$$

3. Были получены следующие свойства коэффициента эффективности проветривания $K$ :

- Коэффициент $K$ принимает постоянное значение для данной конфигурации параметров модели.

- Коэффициент $K$ не зависит ни от расхода входящего свежего воздуха, ни от начальной концентрации вредной примеси, ни от длины зоны отброса газов.

- Коэффициент $K$ зависит от площади сечения выработки (рис. 7) и расстояния от конца трубопровода до забоя (рис. 8), т. е. только от геометрических параметров модели. Результаты моделирования указывают на сильно нелинейную зависимость коэффициента эффективности проветривания $K$ как от сечения проветриваемой выработки, так и от отставания трубопровода от груди забоя, что не позволяет привести простую расчетную формулу для вычисления значения коэффициента $K$ от указанных параметров. Любые изменения сечения выработки, расстояния от конца трубопровода до груди забоя, расположения трубопровода (в центре или с краю), а также сечения воздушной струи будут приводить перестроению структуры крупномасштабных вихрей в большей или меньшей степени, что неизбежно будет отражаться на величине $K$. 


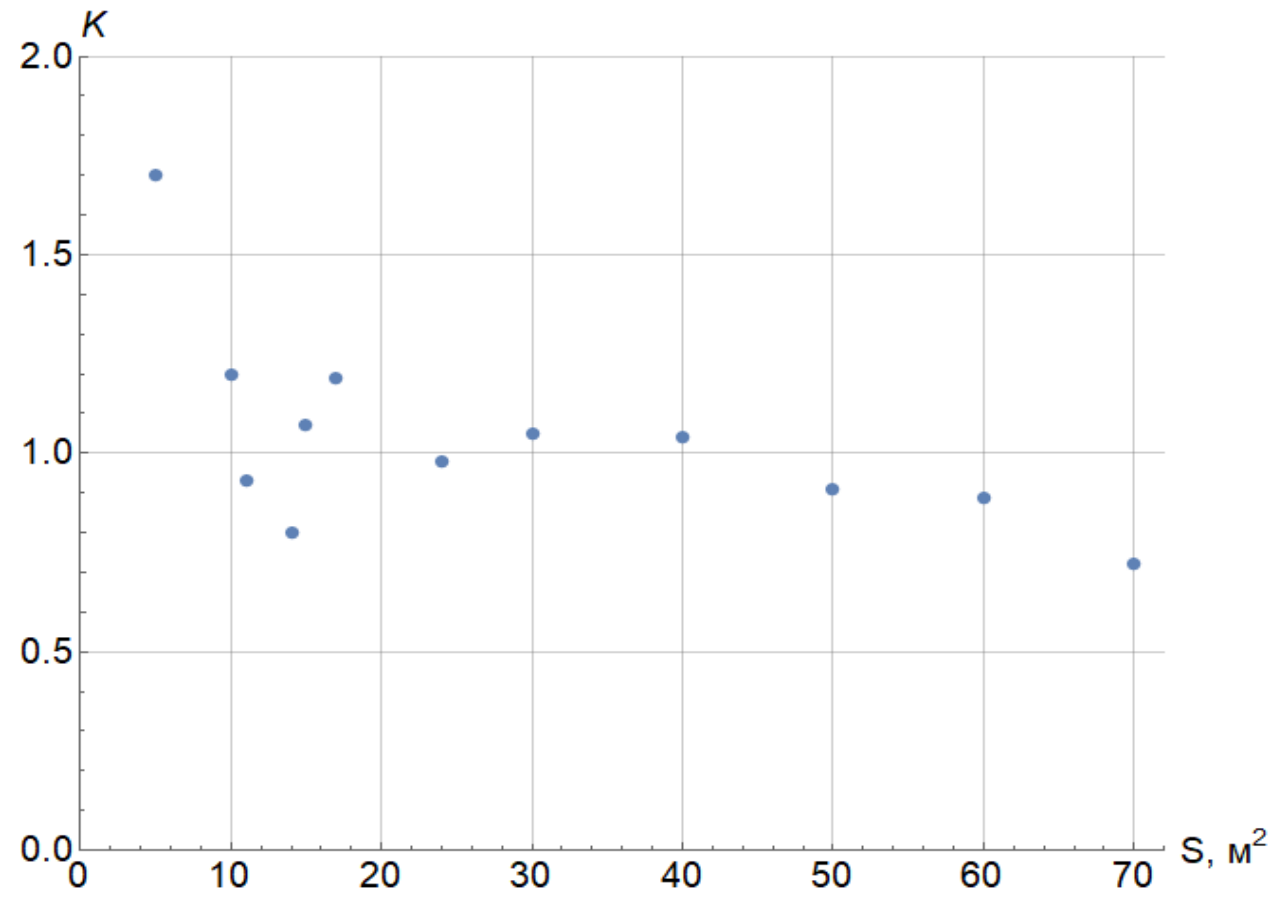

Pис. 7. Зависимость коэффициента эффективности проветривания К от сечения тупиковой выработки при отставании трубопровода $10 \mathrm{M}$

Fig. 7. Ventilation efficiency coefficient $K$ versus cross section area of the dead-end heading relationship. Distance from heading face to air duct is 10 meters

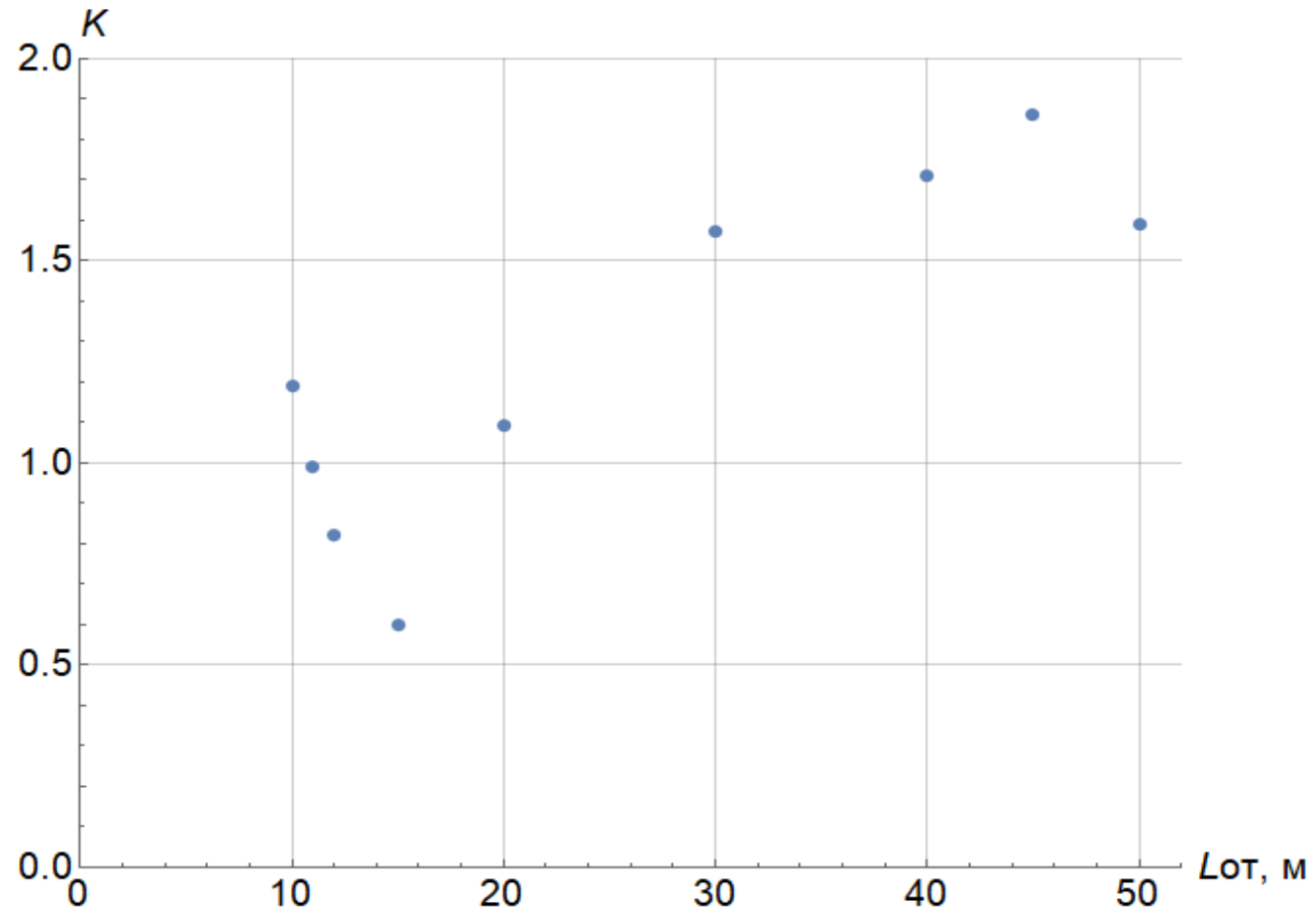

Pис. 8. Зависимость коэффициента эффективности проветривания $K$ от расстояния от конца трубопровода до забоя для выработки сечением $17 \mathrm{M}^{2}$

Fig. 8. Ventilation efficiency coefficient $K$ versus distance from the heading face to the air duct relationship. Cross section area of the dead-end heading is $17 \mathrm{~m}^{2}$

4. Зависимость коэффициента эффективности проветривания $K$ от расстояния от конца трубопровода до забоя, отображенная на рис. 8, указывает на то, что с точки зрения проветривания для выработки сечением $17 \mathrm{~m}^{2}$ наиболее эффективным яв- ляется расстояние 45 м. Для выработок с другим сечением, с другим размером и расположением трубопровода эффективное расстояние будет иметь другое значение. В каждом отдельном случае необходимо корректировать модель и делать 
расчеты с учетом новой конфигурации. Следует отметить, что увеличение расстояния от конца трубопровода до забоя позволит сократить трудозатраты на монтаж трубопровода и ускорит выполнение основных операций по проходке выработки.

5. Поскольку значения коэффициента $K$ для исследуемого пространства параметров лежат в интервале от 0,6 до 1,86, то для инженерных расчетов (например, при оценке времени проветривания выработки после взрывных работ или при расчете количества воздуха, требуемого для проветривания выработки

\section{СПИСОК ЛИТЕРАТУРЫ}

1. Воронин В.Н. Основы рудничной аэро-газодинамики. - М.-Л. Углетехиздат, 1951. - $491 \mathrm{c}$

2. Computational fluid dynamics applied to mining engineering: a review / G. Xu, K.D. Luxbacher, S. Ragab, J. Xu, X. Ding // International Journal of Mining, Reclamation and Environment. 2016. - V. 31. - № 4. - P. 251-275.

3. Tariq F., Bekir G. Evaluation of line brattice length in an empty heading to improve air flow rate at the face using CFD // International Journal of Mining Science and Technology. 2017. - V. 27. - P. 253-259.

4. Conventional and numerical models of blasting gas behaviour in auxiliary ventilation of mining headings / S. Torno, J. Torano, M. Ulecia, C. Allende // Tunnelling and Underground Space Technology. - 2013. - V. 34. - P. 73-81.

5. Studying operational improvements in blast gas clearing using ventilation control / R. Carriere, C. McGuire, E. McLaren, D. Witow // Proceedings of the 16th North American mine ventilation Symposium. - Colorado, USA, 2017. - P. 19.7-19.16.

6. Исследование динамики пылевоздушной смеси при проветривании тупиковой выработки в процессе работы комбайновых комплексов / Л.Ю. Левин, А.Г. Исаевич, М.А. Семин, Р.Р. Газизуллин // Горный журнал. - 2015. - № 1. - С. 72-75.

7. Колесов Е.В. Обоснование последовательного проветривания рабочих зон нескольких тупиковых выработок // Стратегия и процессы освоения георесурсов: сб. науч. тр. - Пермь, 2018. Вып. 16. - С. 291-295.

8. Ушаков К.З. Газовая динамика шахт. - М.: Изд-во МГГУ, 2004. $-481 \mathrm{c}$.

9. Roghanch P., Kocsis K.C. Improving the climatic conditions in development and production workings of hot underground mines by re-designing the auxiliary ventilation system: a case study // International Journal of Mining and Mineral Engineering. 2017. - V. 8 (4). - P. 280-293.

10. Konduri I.M., McPherson M.J., Topuz E. Experimental and numerical modeling of jet fans for auxiliary ventilation in mines / Proceedings of the 6th International Mine Ventilation Symposium. - Pittsburgh, PA, USA, 1997. - P. 505-510. за определенное время) в случае отсутствия исходных данных следует принимать наименышее значение 0,6 , которое соответствует наименее эффективному выносу вредных примесей, что обеспечит дополнительный запас по времени проветривания или по требуемому количеству воздуха для проветривания тупиковой выработки.

Исследование выполнено при финансовой поддержке РФФИ в рамках научного проекта № 19-35-90076.

Работа выполнена при поддержке Программы ФНИ, проект № 0422-2019-0145-C-01.

11. Zhang X., Zhang Y., Tien J.C. The efficiency study of the pushpull ventilation system in underground mine // Proceedings of the 2011 Underground Coal Operators' Conference. - Wollongong, NSW, Australia, 2011. - P. 225-230.

12. Ray R.E., Gilbey M.J., Kumar P. The application of verticallymounted jet fans in ventilation shafts for a rail overbuild // Proceedings of the 12th North American mine ventilation Symposium. - Reno, NV, 2008. - P. 415-424

13. Panigrahi D.C., Mishra D.P. CFD simulations for the selection of an appropriate blade profile for improving energy efficiency in axial flow mine ventilation fans // International Journal of Sustainable Future for Human Security. - 2014. - V. 13 (1). P. $15-21$.

14. Hargreaves D.M., Lowndes I.S. The computational modeling of the ventilation flows within a rapid development drivage // Tunnelling and Underground Space Technology. - 2007. V. 22. - P. 150-160.

15. Silvester S.A. The integration of CFD and VR methods to assist auxiliary ventilation practice. PhD thesis. - Nottingham, 2002. $301 \mathrm{p}$.

16. Auxiliary ventilation in mining roadways driven with roadheaders: validated CFD modelling of dust behavior / J. Torano, S. Torno, M. Menendez, M. Gent // Tunnelling and Underground Space Technology. - 2011. - V. 26. - P. 201-210.

17. CFD simulation of blasting dust for the design of physical barriers / S. Torno, J. Torano, M. Menendez, M. Gent // Environmental Earth Sciences. - 2010. - V. 64. - P. 73-83.

18. Mine face ventilation: a comparison of CFD results against benchmark experiments for the CFD code validation / A.M. Wala, S. Vytla, C.D. Taylor, G. Huang // Mineral Engineering. - 2007. V. 59. - P. 49-55.

19. A practical use of CFD for ventilation of underground works / I. Diego, S. Torno, J. Torano, M. Menendez, G. Malcolm // Tunnelling and Underground Space Technology. - 2011. V. 26. - P. 189-200.

20. Diego I., Torno S., Toraco J. CFD simulation of aerodynamic resistance in underground spaces ventilation // WIT Transactions on The Built Environment. - 2008. - V. 102. - P. 12-23.

Поступила 29.01.2020 г.

\section{Информация об авторах}

Колесов $\boldsymbol{E}$.B., младший научный сотрудник лаборатории математического моделирования геотехнических процессов Горного института УрО РАН.

Казаков Б.П., доктор технических наук, профессор, главный научный сотрудник отдела аэрологии и теплофизики Горного института УрО РАН. 
UDC 622.4

\title{
EFFICIENCY OF VENTILATION OF DEAD-END DEVELOPMENT HEADINGS AFTER BLASTING OPERATIONS
}

\author{
Evgeniy V. Kolesov'1, \\ kolesovev@gmail.com \\ Boris P. Kazakov ${ }^{1}$, \\ aero_kaz@mail.ru \\ ${ }^{1}$ Mining Institute of the Ural Branch of the Russian Academy of Sciences, \\ 78a, Sibirskaya street, Perm, 614007, Russia.
}

The relevance of the research is caused by the need to improve the ventilation system of dead-end headings, labor reduction for their ventilation, speeding up basic mining operations.

The main aim of the research is to determin the effective ventilation parameters of a dead-end heading.

Object of the research is the ventilation system of dead-end heading.

Method of the research is multiparameter three-dimensional numerical simulation based on computational fluid dynamics methods.

The paper introduces the results of three-dimensional numerical simulation of gas dilution in a dead-end heading. The variable parameters of the model are the initial concentration and distribution of toxic gases in the dead-end heading after blasting operations, the distance from the dead-end heading to the air duct, the cross section area of the dead-end heading, as well as the flow rate of fresh air entering through the air duct. The calculation formula for determining the volume of the mixing zone is obtained. It was shown that the airing time of the dead-end heading does not depend on the length of the gas plug, but depends on the initial average concentration of toxic gases in the mixing zone. The values of the dimensionless efficiency coefficient $K$ of a dead-end heading ventilation are obtained. It is shown that the coefficient $K$ is independent on the amount of incoming fresh air flow, and depends only on the geometric parameters of the ventilation system of the dead-end heading and takes a constant value for this configuration of the ventilation system. It is shown that there is an optimal distance from the air duct to the heading face, at which the most effective removal of toxic gases occurs.

\section{Key words:}

Blasting operations, CFD modelling, dead-end heading, ventilation efficiency, mixing zone, gas concentration.

The reported study was funded by RFBR, project number 19-35-90076.

This study was supported by the The Ministry of Science and Higher Education of the Russian Federation as part of a basic research program No. 0422-2019-0145-C-01.

\section{REFERENCES}

1. Voronin V.N. Osnovy rudnichnoy aero-gazodinamiki [Fundamentals of mine aero-gas dynamics]. Moscow; Leningrad, Ugletekhizdat Publ., 1951. 491 p.

2. Xu G., Luxbacher K.D., Ragab S., Xu J., Ding X. Computational fluid dynamics applied to mining engineering: a review. International Journal of Mining, Reclamation and Environment, 2016, vol. 31, no. 4, pp. 251-275.

3. Tariq F., Bekir G. Evaluation of line brattice length in an empty heading to improve air flow rate at the face using CFD. International Journal of Mining Science and Technology, 2017, vol. 27, pp. 253-259.

4. Torno S., Torano J., Ulecia M., Allende C. Conventional and numerical models of blasting gas behaviour in auxiliary ventilation of mining headings. Tunnelling and Underground Space Technology, 2013, vol. 34, pp. 73-81.

5. Carriere R., McGuire C., McLaren E., Witow D. Studying operational improvements in blast gas clearing using ventilation control. Proc. of the $16^{\text {th }}$ North American mine ventilation Symposium. Colorado, USA, 2017. pp. 19.7-19.16.

6. Levin L.Yu., Isaevich A.G., Semin M.A., Gazizullin R.R Issledovanie dinamiki pylevozdushnoy smesi pri provetrivanii tupikovoy vyrabotki $\mathrm{v}$ protsesse raboty kombaynovykh kompleksov [Investigation of the dynamics of the dust-air mixture during ventilation of the dead-end heading during the operation of combine complexes]. Gorny zhurnal, 2015, no. 1, pp. 72-75.

7. Kolesov E.V. Obosnovanie posledovatelnogo provetrivaniya rabochikh zon neskolkikh tupikovykh vyrabotok [Justification of sequential ventilation of several dead-end workings working areas] Strategiya i protsessy osvoeniya georesursov. Sbornik nauchnykh trudov, 2018, vol. 16. pp. 291-295.
8. Ushakov K.Z. Gazovaya dinamika shakht [Gas dynamics of mines]. Moscow, MGGU Publ., 2004. 481 p.

9. Roghanch P., Kocsis K.C. Improving the climatic conditions in development and production workings of hot underground mines by re-designing the auxiliary ventilation system: a case study. International Journal of Mining and Mineral Engineering, 2017, vol. 8 (4), pp. 280-293.

10. Konduri I.M., McPherson M.J., Topuz E. Experimental and numerical modeling of jet fans for auxiliary ventilation in mines. Proc. of the $6^{\text {th }}$ International Mine Ventilation Symposium. Pittsburgh, PA, USA, 1997. pp. 505-510.

11. Zhang X., Zhang Y., Tien J.C. The efficiency study of the pushpull ventilation system in underground mine. Proc. of the 2011 Underground Coal Operators' Conference. Wollongong, NSW, Australia, 2011. pp. 225-230.

12. Ray R.E., Gilbey M.J., Kumar P. The application of verticallymounted jet fans in ventilation shafts for a rail overbuild. Proc. of the $12^{\text {th }}$ North American mine ventilation Symposium. Reno, NV, 2008. pp. 415-424.

13. Panigrahi D.C., Mishra D.P. CFD simulations for the selection of an appropriate blade profile for improving energy efficiency in axial flow mine ventilation fans. International Journal of Sustainable Future for Human Security, 2014, vol. 13 (1), pp. 15-21.

14. Hargreaves D.M., Lowndes I.S. The computational modeling of the ventilation flows within a rapid development drivage. Tunnelling and Underground Space Technology, 2007, vol. 22, pp. $150-160$.

15. Silvester S.A. The integration of CFD and VR methods to assist auxiliary ventilation practice. $\mathrm{PhD}$ thesis. Nottingham, 2002. $301 \mathrm{p}$.

16. Torano J., Torno S., Menendez M., Gent M. Auxiliary ventilation in mining roadways driven with roadheaders: Validated CFD 
modelling of dust behavior. Tunnelling and Underground Space Technology, 2011, vol. 26, pp. 201-210.

17. Torno S., Torano J., Menendez M., Gent M. CFD simulation of blasting dust for the design of physical barriers. Environmental Earth Sciences, 2010, vol. 64, pp. 73-83.

18. Wala A.M., Vytla S., Taylor C.D., Huang G. Mine face ventilation: a comparison of CFD results against benchmark experiments for the CFD code validation. Mineral Engineering, 2007, vol. 59, pp. 49-55.
19. Diego I., Torno S., Torano J., Menendez M., Malcolm G. A practical use of CFD for ventilation of underground works. Tunnelling and Underground Space Technology, 2011, vol. 26, pp. $189-200$.

20. Diego I., Torno S., Toraco J. CFD simulation of aerodynamic resistance in underground spaces ventilation. WIT Transactions on the Built Environment, 2008, vol. 102, pp. 12-23.

Received: 29 January 2020.

\section{Information about the authors}

Evgeniy V. Kolesov, junior researcher, Mining Institute of the Ural Branch of the Russian Academy of Sciences.

Boris P. Kazakov, Dr. Sc., professor, chief researcher, Mining Institute of the Ural Branch of the Russian Academy of Sciences. 